\title{
A BIAXIAL MICROMIRROR FOR ANGULAR AND DEPTH SCANNING IN OPTICAL COHERENCE TOMOGRAPHY (OCT)
}

\author{
U. Izhar ${ }^{1 *}$, B.S. Ooi ${ }^{2}$, and S.Tatic-Lucic ${ }^{l}$
}

${ }^{1}$ Sherman Fairchild Center, Electrical \& Computer Engineering Department, Lehigh University, Bethlehem, PA 18015, USA

${ }^{2}$ Division of Physical Sciences and Engineering, King Abdullah University of Science and Technology (KAUST), Thuwal, KSA

\begin{abstract}
A novel gimbal-less, thermally actuated micromirror is developed which can be used as both a reference and scanning mirror in optical coherence tomography (OCT) applications. Maximum deflection angle of up to $28^{\circ}$ has been achieved at $12 \mathrm{~mW}$ in scanning mode. A vertical displacement of $116 \mu \mathrm{m}$ at $12 \mathrm{~mW}\left(78 \pm 4^{\circ} \mathrm{C}\right)$ was observed in piston mode. Our design goal for this mirror is to undergo $5^{\circ}$ angle. This deflection was accomplished at a temperature increase of $17 \pm 5^{\circ} \mathrm{C}$ with respect to ambient. Our device can be integrated with GaAs optics due to this moderate temperature increase during its operation.
\end{abstract}

\section{INTRODUCTION}

Multi-axis micromirrors have been widely reported in different design topologies with different actuation methods, such as electrostatic, electromagnetic, piezoelectric and electrothermal. These micromirrors are used in various applications including biomedical imaging, optical switching, beam positioning, and displays.

Zara and Smith [1] demonstrated an electrostatic design where a single axis micromirror was described. Angular displacement close to $30^{\circ}$ at a voltage of $90 \mathrm{~V}$ was reported in this work. An electromagnetic design was proposed in [2] with a reported angular displacement of $45^{\circ}$ at an input current of $450 \mathrm{~mA}$ in [2]. Similarly, a piezoelectric actuation scheme was used by Kim et. al. in [3] resulting in angular displacement of up to $6.1^{\circ}$ at $16 \mathrm{~V}$. Finally, many electrothermal micromirrors have been reported for scanning applications. A typical example is shown by Buhler et. al. [4] and this micromirror used bimorph actuation To achieve a rotation angle of $5^{\circ}$ for an applied power of $5 \mathrm{~mW}$. Later, Lammel et. al. [5] reported rotation angles of up to $30-40^{\circ}$ for applied power of $15 \mathrm{~mW}$. Recently, electrothermal designs that were used in OCT application were reported by Xie et. al. [6] and Singh et al. [7]. In latter work, an angle of $17^{\circ}$ was achieved with the temperature of $90^{\circ} \mathrm{C}$ in the actuator with applied power of approximately $37 \mathrm{~mW}$ [7].

In this paper, we propose a multi-axial design that can achieve large angular deflection of up to $28^{\circ}$ at low power of $12 \mathrm{~mW}$. The proposed design results in modest temperature increase of $78 \pm 4^{\circ} \mathrm{C}$ with respect to ambient during the operation at maximum angular deflection.

\section{MICROMIRROR DEVICE LAYOUT AND OPERATION}

The micromirror device consists of a $3 \mu \mathrm{m}$ thick single crystal silicon mirror with a $1 \mu \mathrm{m}$ thick aluminum reflective coating on it. The mirror is connected to four electrothermal actuators (ETAs) through four $1.5 \mu \mathrm{m}$ thick undoped polysilicon flexural connectors, see Figure 1. The electrothermal actuators consist of $1.5 \mu \mathrm{m}$ thick undoped polysilicon as their lower structural layer and $1 \mu \mathrm{m}$ thick aluminum as their upper layer. When a voltage is applied to the heater contact pads, the actuator undergoes vertical displacement due to Joule heating. This motion is then transferred to the mirror through a corresponding flexural connector.

Our envisioned system-on-chip OCT system setup is also shown in Figure 1. The chip includes integrated waveguides
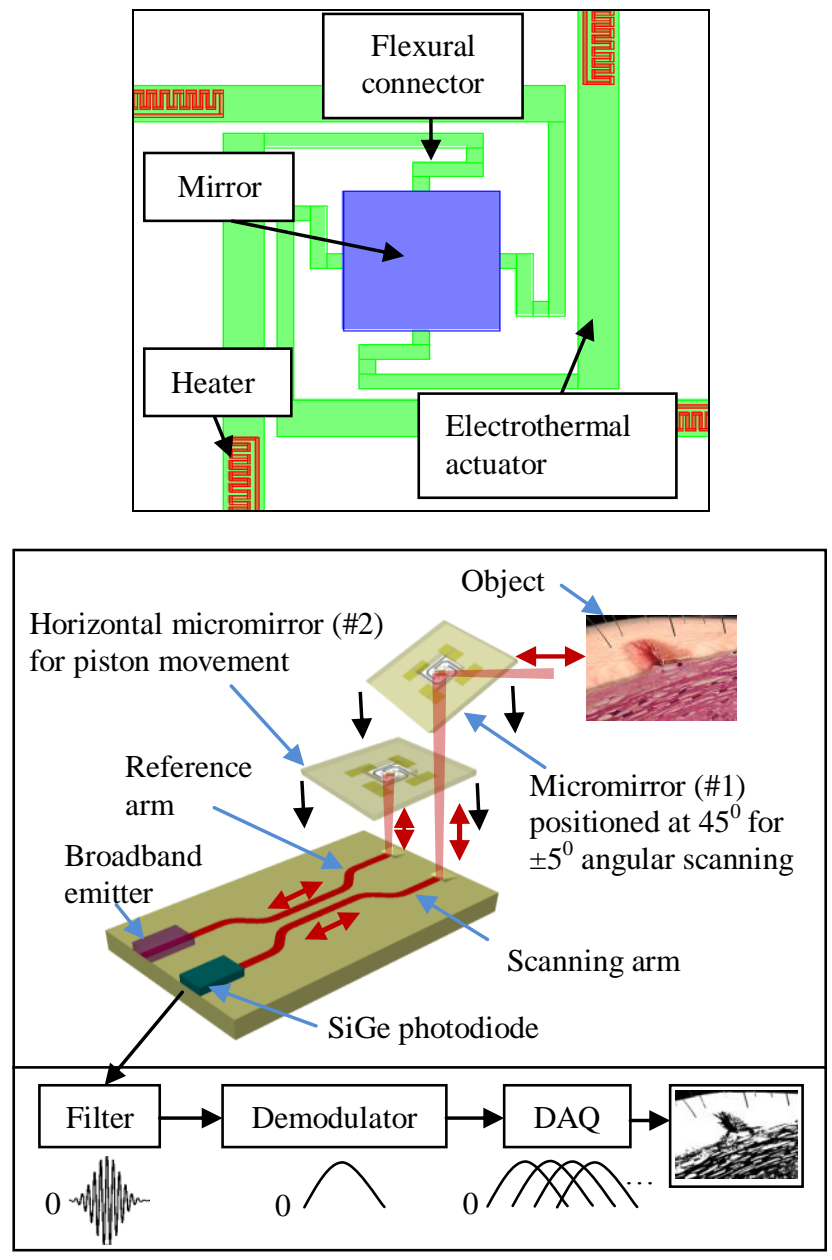

Figure 1: (Top) Schematic of the proposed micromirror design, (Bottom) $3 D$ representation of envisaged integrated (miniature) OCT system

for the reference and sample arms of the interferometer, a broadband light source and photo diode. The proposed micromirror would be integrated horizontally for depth scanning (piston motion) at the end of reference arm, whereas the micromirror would be positioned at an angle of $45^{\circ}$ (to direct light to and from the target sample) at the end of sample arm. The broadband light source will be monolithically integrated with the device. A silicon germanium photo diode would be used in the return path to detect the intensity of the incoming interference signal in time-domain. This time varying signal usually has a DC offset which can be removed by the bandpass filter. The signal will be demodulated for the envelope retrieval of the interference pattern. The signal can then be sampled by a data acquisition (DAQ) card and after processing the corresponding image can be displayed.

For feasible integration, temperature increase and power dissipation must be limited. Several design parameters were developed as goals for our micromirror device and are listed in Table 1. 
Table 1: Design goals for MEMS micromirror

\begin{tabular}{ll}
\hline Design Property & Specification \\
\hline Mirror Scanning Axis & 2 Axes \\
Mirror Scanning Angle & $+/-5$ degrees \\
Temperature Increase & $<20$ degrees C \\
Mirror Actuation Voltage & $<15 \mathrm{~V}$ \\
Mirror Actuation Frequency & $\geq 4 \mathrm{~Hz}$ \\
\hline
\end{tabular}

According to Table 1, minimum out-of-plane displacement of \pm 5 degrees and maximum temperature increase of $20 \mathrm{deg}$. $\mathrm{C}$ are desired. These initial goals do not require the mirror to undergo vertical displacement. However, for a complete OCT setup a piston motion in the reference arm is required for depth perception of the target. For this reason, the device was designed such that it has dual functionality.

The thermo-physical behavior of the bimorph device (i.e. the change in curvature with the increase in temperature), when width of the actuator is constant can be given as [8]:

$$
\frac{1}{r}=\frac{6\left(t_{1}+t_{2}\right)(\Delta \alpha)(\Delta T)}{t_{1} t_{2}\left[\epsilon \tau^{2}+\frac{1}{\epsilon \tau^{2}}+4 \tau+\frac{4}{\tau}+6\right]}
$$

where $r$ is the curvature, $t_{1}$ and $t_{2}$ are the thicknesses of upper and lower layers respectively. Parameter $\Delta \alpha$ is the difference in coefficients of thermal expansion between the two layers and $\Delta T$ is the temperature increase from ambient. In the equation above, $\tau=\frac{t_{1}}{t_{2}}, \epsilon=\frac{E_{1}}{E_{2}}$ and $E_{1}$ and $E_{2}$ represents the Young's modulus of upper and lower layers respectively.

Moreover, if the convection and radiation are neglected, the temperature increase in the heater with applied power can be given as:

$$
\Delta T=P_{e} R_{t}=\left(\frac{V^{2}}{R_{e}}\right) R_{t}
$$

where $R_{t}=\frac{l}{\kappa w t}$ is the thermal resistance, $\kappa$ is thermal conductivity, and $\mathrm{l}, \mathrm{w}$, and $\mathrm{t}$ are length, width and thickness of the heater respectively.

Optimal thickness ratio of the actuator layers was found out to be 0.707 [9] which gives $1 \mu \mathrm{m}$ thick upper aluminium layer for a $1.5 \mu \mathrm{m}$ thick lower polysilicon structural layer.

\section{FINITE ELEMENT ANALYSIS}

Finite element analysis of the device was carried out using MEMS specific software, CoventorWare ${ }^{\mathrm{TM}}$ (Coventor, Inc.). Static analysis was performed using the software's electro-thermomechanical solver.

In the simulation model, the silicon substrate was covered with an insulating layer of oxide above it. Actuators were anchored on the oxide layer of the substrate. The bottom face of the substrate was assigned a fixed mechanical boundary condition and a thermal boundary condition of $300 \mathrm{~K}$ (ambient temperature). Effects from convection and radiation were also considered from exposed aluminium surfaces. Though convection and radiation boundary conditions were later found to have less than $1 \%$ effect on the results, they were included in the analysis presented here. A bias was applied to one of the actuators to simulate the static deflection of the mirror.

The flexibility of design options in the software was used to observe the response of the mirror device for different actuator lengths $(500 \mu \mathrm{m}$ and $550 \mu \mathrm{m})$, heater positions, and flexural connector shapes. For the heater, it was found that its position affects the amount of power required to displace the actuator. If the heater is positioned solely on the anchor (over the substrate), it requires $333 \%$ more power to introduce the same temperature increase than the heater positioned on the substrate and extended into the actuator. This can be due to the fact that most of the heat flux is used to increase the temperature of the substrate which acts as temperature sink. However, when the heater is extended into the actuator, the heat flux encounters a much smaller mass compared to the substrate and thus the temperature along its length increases.

The flexural connector width and length was also studied as it relates to the flexibility and the amount of force that can be exerted on a mirror edge. Angular displacement and temperature increase observed from the analysis are presented in Figure 2. It can be seen from the figure that according to simulations, angular displacement of 5 degrees can be achieved at $2 \mathrm{~mW}$ for actuator length of $550 \mu \mathrm{m}$ and at $4.5 \mathrm{~mW}$ for actuator length of $500 \mu \mathrm{m}$.

The temperatures at these angular displacements are $18^{\circ} \mathrm{C}$ for $550 \mu \mathrm{m}$ and $32^{\circ} \mathrm{C}$ for $500 \mu \mathrm{m}$. Though the temperature is slightly higher in the latter design than was expected, it is worth noticing
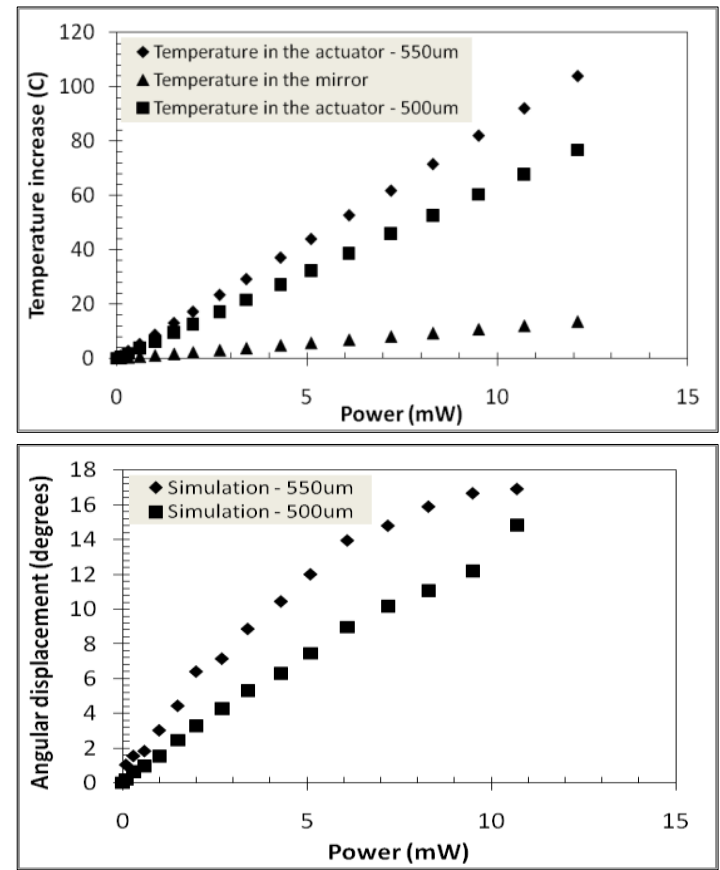

Figure 2: (Top) Simulated temperature increase from the ambient with applied power, (Bottom) Simulated angular displacement of $500 \mu \mathrm{m}$ and 550 $\mathrm{m}$ long actuators with the applied power

here that the simulations depicted the substrate temperature increase much lower $\left(<5^{\circ} \mathrm{C}\right)$ than in the actuator. As the substrate (not the actuator) would be in contact with optics this temperature increase would not affect the integration. Moreover, according to this simulation the temperature of the mirror was much lower than the temperature of the actuator during operation. This low temperature on the mirror ensured that there was no significant curvature change in the mirror due to temperature increase. After the preliminary feasibility of the device was studied through this analysis, actual fabrication was carried out.

\section{DEVICE FABRICATION}

The fabrication process was carried out in Cornell's Nanofabrication Facility (CNF). The six mask fabrication process started with a four inch $(100 \mathrm{~mm})$ diameter, silicon on insulator 
(SOI) wafer with $3 \mu \mathrm{m}$ thick p-type device layer (boron-doped, $100 \Omega \mathrm{cm}$ ), $2 \mu \mathrm{m}$ oxide layer and $500 \mu \mathrm{m}$ handle wafer (Ultrasil Corp. CA). The first step in the process was to define $3 \mu \mathrm{m}$ thick mirror base in the wafer device layer for support of aluminum mirror. Deep reactive ion etching (DRIE) using UNAXIS 770 ICP etcher was used for this purpose. Second, an undoped polysilicon layer was deposited using low pressure chemical vapor deposition (LPCVD) in two steps to embed the platinum heater. In the first step, $1 \mu \mathrm{m}$ thick layer (@ $590^{\circ} \mathrm{C}, 150 \mathrm{sccm} \mathrm{SiH}_{4}$ ) was deposited. Before the second step, a $0.1 \mu \mathrm{m}$ thick platinum was evaporated using e-beam evaporator, SC4500 (CVC Inc.) and patterned by lift off in Microposit 1165 stripper (Shipley). A 50nm thick titanium layer was used under the platinum for better adhesion. The second step was deposition of $0.5 \mu \mathrm{m}$ thick polysilicon (@ 590 $\mathrm{C}, 150$ sccm $\mathrm{SiH}_{4}$ ) that followed after defining the heater.

To fabricate the upper layer of actuator and mirror, $1 \mu \mathrm{m}$ thick layer of aluminum was evaporated using e-beam and patterned using lift-off in Microposit 1165. Next, polysilicon was etched using reactive ion etching (RIE) in an Oxford 80 etcher (Plasma $\mathrm{Lab}$ ) to define actuators, and flexural connectors with aluminum as a mask. Following this, aluminum was removed from the flexural connectors and contact pads using wet etching. The whole front side fabrication was completed by removing the $0.5 \mu \mathrm{m}$ thick polysilicon layer from the platinum contact pads using $\mathrm{SF}_{6} / \mathrm{O}_{2}(30$ sec.) and $\mathrm{CF}_{4}(150 \mathrm{sec}$.) to expose the electrical contacts.

To release the device, dry etch (instead of wet $\mathrm{KOH}$ etch) was selected, as it needs less space for opening windows. One micron thick plasma enhanced chemical vapor deposition (PECVD) oxide and $7 \mu \mathrm{m}$ of photoresist (SPR 220-7) were used as masks for backside etching of the $500 \mu \mathrm{m}$ handle wafer. DRIE etch was carried out to remove all $500 \mu \mathrm{m}$ of the handle wafer with 30 minute cool-down cycles between $50 \mu \mathrm{m}$ intervals to get better sidewall profiles. The last step in the release process was oxide etch. Dry etching was used again with $\mathrm{CHF}_{3} / \mathrm{O}_{2}(50 \mathrm{sccm} / 2 \mathrm{sccm}$, $240 \mathrm{~W}$ ) for this purpose as it is less violent than the wet etch, in which the fluid pressure can break the released structures. The process flow diagram is given in Figure 3 and an optical micrograph of the released device is shown in Figure 4.
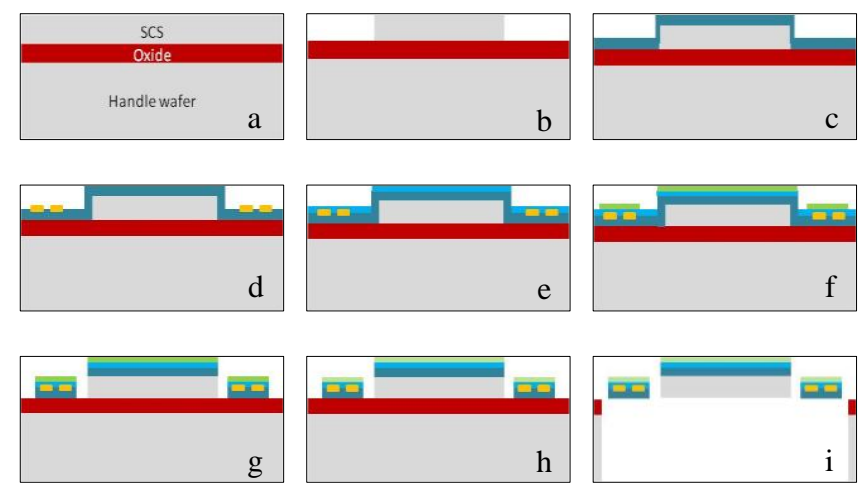

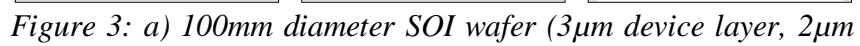
oxide layer), b) photolithography and deep reactive ion etching (DRIE) to define the mirror base, c) LPCVD of first portion of polysilicon (1 $\mu \mathrm{m})$, d) E-beam evaporation of titanium(Ti) and platinum(Pt) and patterning by lift-off (100nm), e) LPCVD of second portion of polysilicon to embed the platinum resistor (500nm), f) E-beam evaporation of aluminum (Al) and patterning by lift-off (1 $1 \mu \mathrm{m}), \mathrm{g})$ RIE of polysilicon using aluminum as a mask, h) etching of aluminum and second portion of polysilicon to expose the platinum contact pads, i) DRIE of silicon and RIE of oxide from backside to release the device

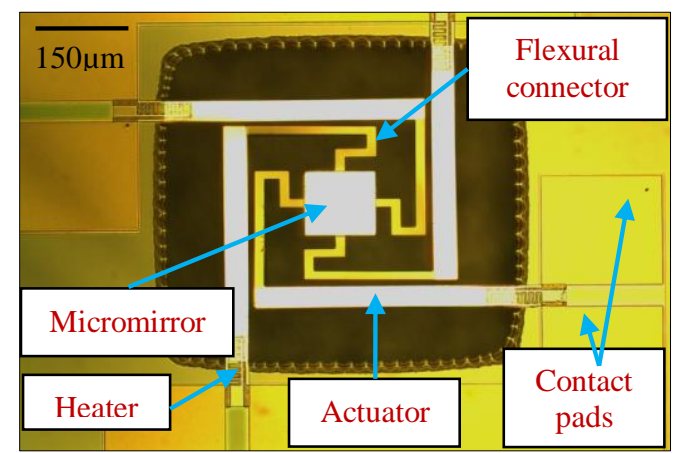

Figure 4: Optical micrograph of a released micromirror device

\section{CHARACTERIZATION AND DISCUSSION}

Angular displacement for scanning arm application

First, we performed measurements for the angular displacement of the mirror. A $633 \mathrm{~nm}$ HeNe red laser was placed perpendicular to the mirror plane with light incident vertically down on the mirror. A millimeter scaled screen was placed $55 \mathrm{~mm}$ above the mirror. To pass the light from the screen, a pinhole in the screen was created. The angular displacement of the mirror was then calculated by observing the displacement of laser spot on the screen, see Figure 5 (Top). The standard deviation of each mean value measured for five device samples is also shown. The maximum angles of $23^{\circ}$, and $28^{\circ}$ were measured at $12 \mathrm{~mW}$ for $500 \mu \mathrm{m}$ and $550 \mu \mathrm{m}$ long actuator designs respectively.

These results were better than what we predicted through simulations (Figure 2) and yielded higher angular displacements than anticipated. This is believed to be due to rotation or vertical displacement of the mirror introduced by the stiffness of adjacent actuators and flexural connectors. Simulations were repeated with taking the vertical displacement into account. The results are shown in Figure 5 (bottom).
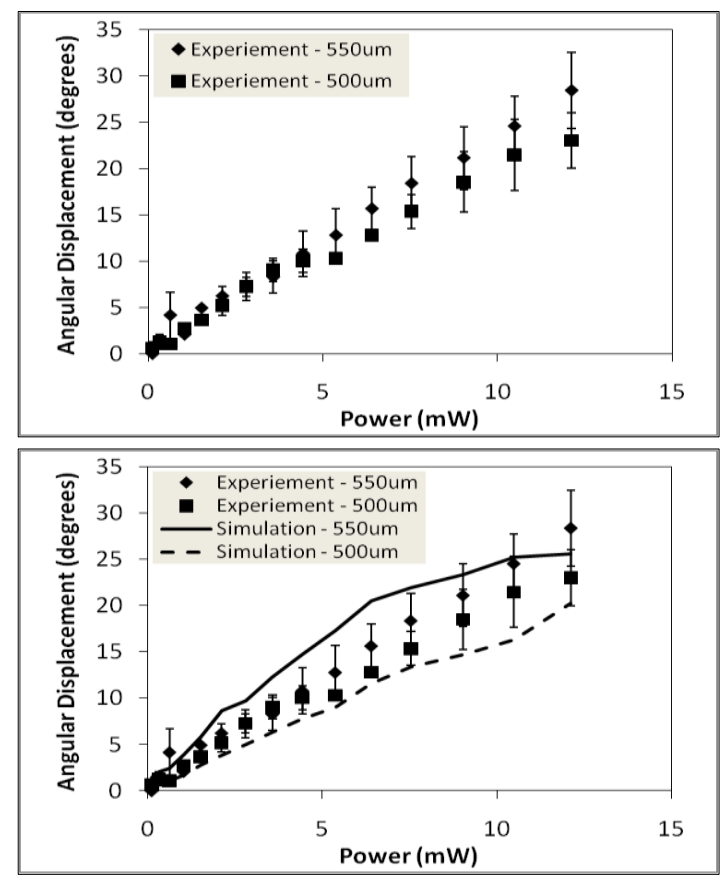

Figure 5: (Top) Angular displacement as a function of applied power, (Bottom) Comparison between angular displacements acquired with experiment and simulations taking into account the undesirable vertical displacement of the mirror in scanning mode 
It can be seen that the angular displacement from simulation and experiment shows better agreement with an average difference of $23 \%$ between the values.

\section{Electrothermal response}

As stated earlier, for the integration of the mirror in future with the miniaturized OCT system it is critical that the temperature increase in the device should remain low. For this purpose, we experimentally determined the temperature increase by measuring the change in resistance of the heater as a function of input power and is shown in Figure 6 . The average error of the mean value from simulation is $19 \%$.

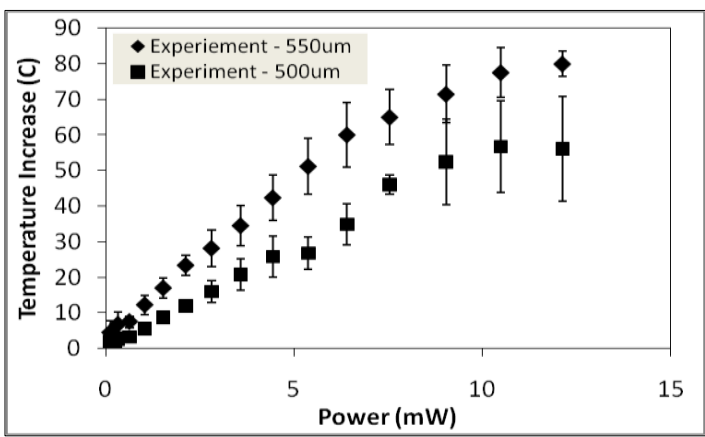

Figure 6: Temperature increase from ambient as a function of the applied power

\section{Vertical displacement for reference arm application}

As our device has dual functionality, it was also characterized for vertical displacement using optical profilometer. The results are presented in Figure 7 and show that a vertical displacement of up to $64 \mu \mathrm{m}$ for $500 \mu \mathrm{m}$ and $116 \mu \mathrm{m}$ for $550 \mu \mathrm{m}$ long actuators can be achieved for a power input of $12 \mathrm{~mW}$ with a temperature increase of $57 \pm 14 \mathrm{deg}$. $\mathrm{C}$ and $78 \pm 4 \mathrm{deg}$. C respectively (Figure 6). The results are compared with analytical calculations (using Equation 1). It can be seen that the difference between the two results lie between the standard deviation of the mean experimental values.

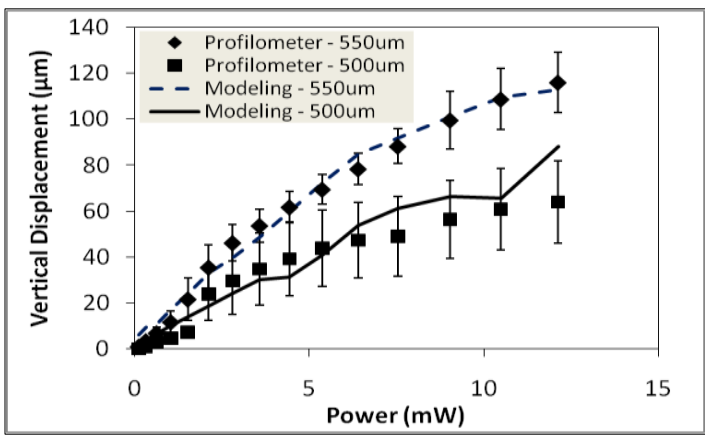

Figure 7: Comparison between vertical displacement (of different mirror designs) measured using profilometer with values calculated using Equation 1

\section{Transient response}

The frequency response of the mirror was characterized to measure the minimum and maximum time it would take for one scan and it is shown in Figure 8. It was found out that the output value falls to $70 \%$ of its value at $10 \mathrm{~Hz}$.

Curvature and roughness were measured as they affect the focus depth in OCT imaging. For the current mirror, the curvature was found out to be $2.4 \mathrm{~mm} \pm 0.26 \mathrm{~mm}$. This curvature is significant. It is believed to be due to the insufficient thickness of the silicon

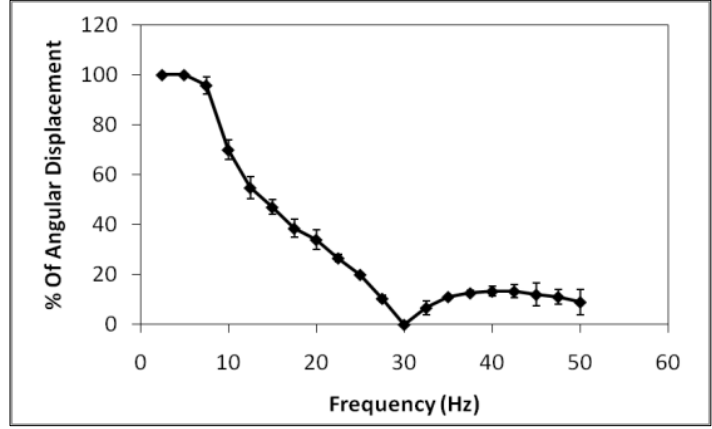

Figure 8: Transient response of the mirror device

supporting layer $(3 \mu \mathrm{m})$ beneath the $1 \mu \mathrm{m}$ reflective aluminum layer. Moreover, there can be mismatch at the interface of polysilicon layer which is deposited in two steps. The curvature can be improved by increasing the thickness of supporting single crystal silicon, decreasing the aluminum thickness and removing any sandwich layer between the two. The roughness of the mirror was found to be $100 \mathrm{~nm} \pm 20 \mathrm{~nm}$.

\section{CONCLUSION}

We have presented, for the first time, an electrothermallyactuated, gimbal-less, multi-axis mirror which has the capability to operate in both scanning and piston mode and to achieve high angular (up to $28^{0}$ ) and vertical displacements $(116 \mu \mathrm{m})$ at low powers of $12 \mathrm{~mW}$ and voltages of up to $6 \mathrm{~V}$. The temperature increase at this maximum angular and vertical displacement is $78 \pm 4$ deg. C. Our original specification of $\pm 5^{\circ}$ scanning angle can be achieved at a low power of $2 \mathrm{~mW}$ and at a maximum temperature increase of $17^{\circ} \mathrm{C} \pm 5^{\circ} \mathrm{C}$ from ambient. The temperature increase of the substrate is less than $5^{\circ} \mathrm{C}$ for this input. These properties make it compatible with other integrated optics to realize miniature OCT imaging. Currently, we are testing the mirror in a benchtop OCT system and experiments to get one dimensional intensity profiles are underway.

\section{ACKNOWLEDGEMENTS}

This work was supported by PITA (Pennsylvania Infrastructure Technology Alliance), PITA-743-07 and the fabrication was carried out in CNF (Cornell Nanoscale Science and Technology Facility) at Cornell University.

\section{REFERENCES}

[1]. J. Zara, S. Smith, Sens. and Act. A 102 (2002), p. 167-174.

[2]. J. W. Judy, R. S. Muller, JMM 63 (1997), p 249-256.

[3]. S. J. Kim, Y. H. Cho, H. J. Nam and J. U. Bu, JMM 18 (2008), p 1-7.

[4]. J. Bühler, J. Funk, O. Paul, F.-P. Steiner, H. Baltes, Sens. and Act. A 46-47 (1995), p 572-575.

[5]. G. Lammel, S. Schweizer, P. Renaud, Kluwer Academic, Boston, 2002.

[6]. A. Jain, S. T. Todd, G. K. Fedder, H. Xie, 2003 OSA Annual Meeting, Tucson, AZ, October 2003.

[7]. J. Singh, J.H.S. Teo, Y. Xu, C.S. Premchandran, N. Chen, JMM, 18 (2008) p. 1-9.

[8]. C. Liu,"Foundaions of MEMS",Pearson Prentice Hall (2006).

[9]. W. Peng, Z. Xiao, and K. R. Farmer, Proceedings of the 2003 Nanotechnology Conference, CA, 1 (2003), p. 376-379.

\section{CONTACT}

*U. Izhar, tel: +1-610-758-2003; umi205@lehigh.edu 